\title{
Introduction to the special issue on software technologies for embedded and ubiquitous systems
}

\author{
Lynn Choi • Raimund Kirner
}

Published online: 14 December 2014

(C) Springer-Verlag Wien 2014

Ubiquitous embedded systems are one of the technology drivers to improve our daily life, much more than we normally recognize. To engineer such systems we have to overcome many challenges. For example, such systems typically have to support low-power network connection with nearby computing devices in the context of high mobility, while at the same time incorporating the latest hardware developments in order to allow for cost-effective yet powerful systems.

The following five articles in this special issue cover two important issues of software technologies for embedded and ubiquitous systems: safety critical systems and wireless networking technologies and applications.

LINKORD: Link Ordering Based Collection Tree Protocol for Wireless Sensor Networks

In this article Radi et al. propose an improved collection tree protocol for wireless networks with multi-hop communication pattern. This new protocol improves reliability and reduces energy consumption.

\section{Influenza Surveillance and Forecast with Smartphone Sensors}

In this article Lee et al. describe a method to improve the prediction of influenza proliferation and to suggest an improved vaccination procedure based on sensor data collected from smartphone users.

L. Choi

School of Electrical Engineering, Korea University, Seoul, The Republic of Korea e-mail: 1choi@korea.ac.kr

R. Kirner $(\bowtie)$

School of Computer Science, University of Hertfordshire, Hatfield, UK

e-mail: r.kirner@herts.ac.uk 
Reasonability of MC/DC for Safety-Relevant Software Implemented in Programming Languages with Short-Circuit Evaluation

In this article Kandl investigates whether the modified condition/decision coverage (MC/DC) criterion is adequate for safety-related software, concluding that multiple condition coverage (MCC) shows the best result with minimal cost.

Light-weight Synchronous Java (SJL)—An Approach for Programming Deterministic Reactive Systems with Java

In this article Motika and von Hanxleden describe a Light-weight synchronous Java, a language based on Java, but extended with concurrency mechanisms developed with synchronous programming languages.

Filter-based Address Auto Configuration Protocol (FAACP) for Duplicate Address Detection and Recovery in MANETs

In this article Reshmi and Murugan address the auto-configuration of mobile adhoc networks (MANETs) by proposing a scalable address assignment protocol. The protocol can cope well with the dynamic changes happening in MANETs. 